\title{
INFLUENCIAS DEL CLIMA FAMILIAR. ESTRATEGIAS DE APRENDIZAJE E INTELIGENCIA EMOCIONAL EN EL RENDIMIENTO ACADÉMICO.
}

\author{
Dra. Amparo Sotil Brioso \\ Mag. Alberto Quintana Peña
}

\begin{abstract}
El presente trabajo tiene como finalidad hacer un estudio empírico dc la contribución de los efectos combinados del clima familiar las estrategias de aprendizaje y la inteligencia emocional sobre el rendimiento académico investigación realizada en una muestra de 250 estudiantes de la Facultad de Psicología de la Universidad Nacional Mayor de San Marcos. Encontrando como resultado que en cuanto al Clima Familiar predominan las familias de rango medio y balanceadas mientras que respecto a la inteligencia emocional, se observa una capacidad emocional por debajo del promedio en los componentes intra personales. Por ultimo es de resaltar que si bien existe una correlación estadísticamente significativa entre los tipos de familia, la inteligencia emocional y las estrategias de aprendizaje. Sin embargo, entre estas variables y el rendimiento académico evaluado a través del promedio ponderado de las calificaciones obtenidas por los estudiantes durante su permanencia en la universidad, no existe una correlación estadística significativa.
\end{abstract}

PALABRAS CLAVE: Universitarios, Clima Familiar, Inteligencia Emocional, Estrategias de aprendizaje, Rendimiento Académico.

"The present work has as purpose to make an empiric study of the contribution of the combined effects of the family climate, the learning strategies and the emotional intelligence on the academic yield investigation carried out in a sample of 250 students of the Ability of Psychology of the National University bigger than San Marcos. Finding as a result that, as for the Family Climate the hall and balanced range families prevail, while regarding the emotional intelligence, an emotional capacity is observed below the average in the component personal intra. For I finish. it is of standing out that although a correlation exists statistically significant among the family types, the emotional intelligence and the learning strategies however, between these variables and the academic yield evaluated through the pondered average of the qualifications obtained by the students during its permanency in the university, a significant statistical correlation doesn't exist.

KEY WORDS: University students, Family Climate, Emotional Intelligence, learning Strategies, Academic Yield. 


\section{INTRODUCCIÓN}

Desde el punto de vista de las relaciones humanas, la familia es el centro y núcleo de la sociedad, cuyo papel primordial en el proceso de socialización es el establecimiento de normas, reglas y sobre todo valores éticos y morales. Sin embargo, el modelo económico que ha venido implementándose en el país en los últimos años distancia a los jefes de familia de esta responsabilidad, ya que ambos tienen que trabajar a fin de satisfacer las necesidades materiales de sus hijos, descuidando un aspecto tan importante como es el factor emocional el cual es la base de la formación de la personalidad de niños y adolescentes, (Benites.1998). Al mismo tiempo, la mayoría de investigaciones realizadas en nuestro país señalan la importancia que tiene la familia como factor de riesgo o de debilidad psicosocial para la aparición en los niños y adolescentes de problemas sociales, interpersonales, educativos y emocionales. Los estudios sobre estructura familiar, familia desintegrada contingencias familiares, clima social familiar y Crisis familiares así lo demuestran (Benites 1998).

De esta suerte resulta necesario plantear la siguiente interrogante:

¿En qué medida el clima familiar se relaciona con los estilos de aprendizaje, la inteligencia emocional y el rendimiento académico de los estudiantes de la Facultad de Psicología de la U.N.M.S.M?

Para profundizar la investigación de estos hechos en el ámbito de la educación superior, en el presente estudio se ha adoptado la concepción sistémica de la familia, en particular, el Modelo Circumflejo de Olson (1979) sobre el clima familiar (Olson, Russell, y Sprenkle, 1979, 1980, 1982 y 1983). El modelo Circunflejo evalúa dos dimensiones del comportamiento familiar, a saber: Cohesión y Adaptabilidad.

La cohesión familiar examina el grado en que los miembros de la familia están separados o conectados a ella. Es el vínculo emocional que los miembros de la familia tienen entre sí.

Se distinguen 4 niveles de cohesión que varían desde desligada (muy baja cohesión), separada (baja a moderada) conectada (moderada a alta) y amalgamada (muy alta) (Figura 1). Se sostiene que los niveles centrales de cohesión (separada y conectada) son los que dan lugar a un óptimo funcionamiento familiar. En cambio los niveles extremos (desligada y amalgamada) son generalmente vistos como problemáticos.

La adaptabilidad familiar se relaciona con la flexibilidad del sistema familiar. es decir con la capacidad de cambio de la familia frente a nuevas situaciones. Se define como la habilidad de un sistema marital o familiar para cambiar su estructura de poder y las relaciones de roles, en respuesta al estrés situacional o al desafió al que se enfrenta a través de su desarrollo.

Se distinguen 4 niveles de adaptabilidad que van desde rígida "(muy baja), estructurada (baja a moderada), flexible (moderada a alta) y caótica (muy alta). (Figura 1).

Basándonos en el modelo Circumplejo, es de esperar que los niveles muy altos de adaptabilidad (caótico) y los niveles muy bajos (rígido) puedan ser problemáticos influyendo en los estilos de aprendizaje, la inteligencia emocional y, por ende, afectando al rendimiento académico de los estudiantes universitarios. En cambio cuando las relaciones son estructuradas y flexibles denotarían un mayor equilibrio frente al cambio y una estabilidad más funcional" lo que podría incidir de modo satisfactorio en las estrategias de 
aprendizaje y la inteligencia emocional, y por ende, influyendo positivamente en los logros académicos.

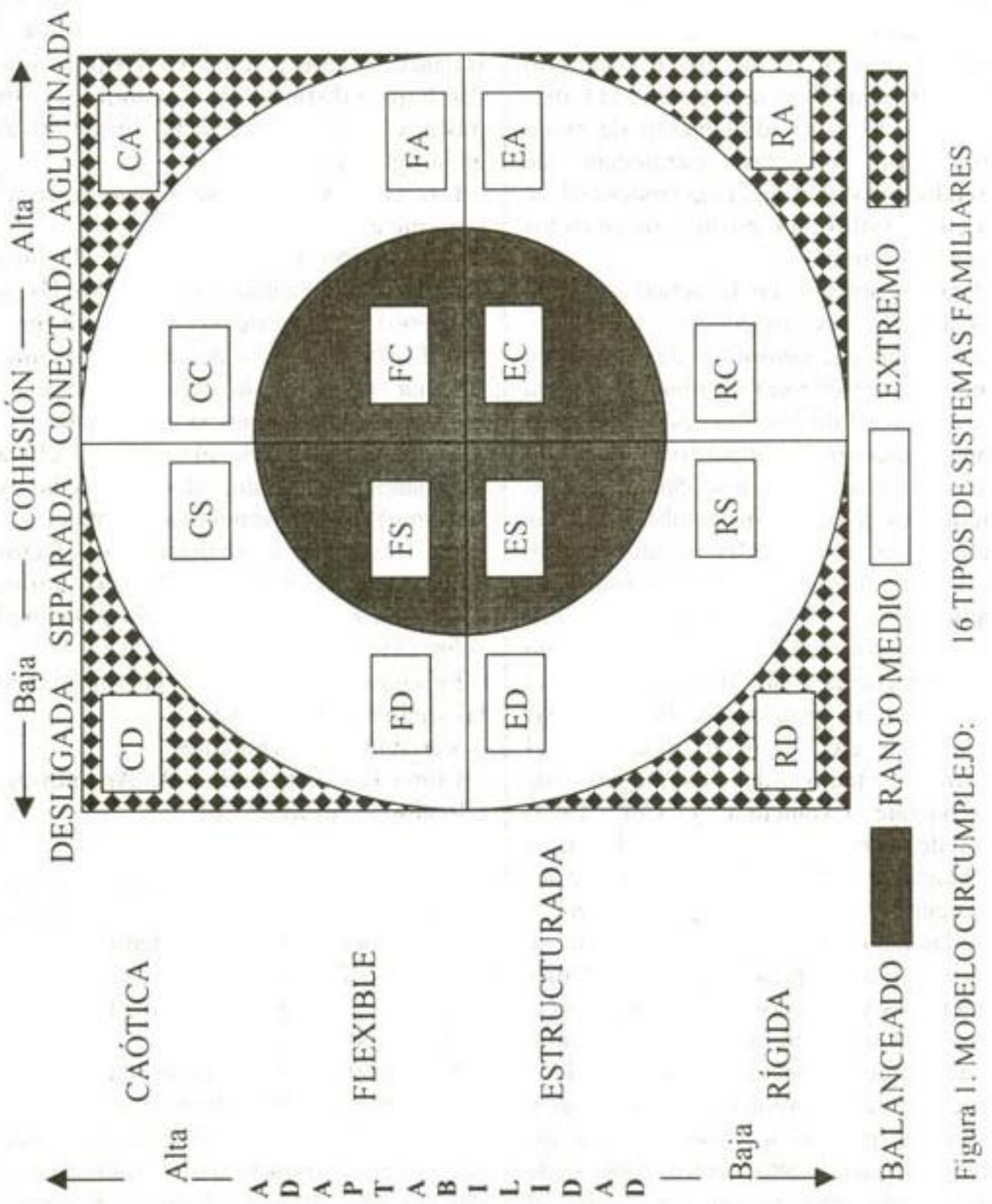


Paradójicamente, en la actual sociedad globalizada, las exigencias científico tecnológicas del entorno y de la vida en general hacen que sea un imperativo para el éxito social de los jóvenes el que éstos sean productivos y competitivos, haciendo necesario que las capacidades dc los estudiantes abarquen más ámbitos que las tradicionales capacidades de abstracción, de lógica formal, de comprensión de implicaciones complejas y de amplios conocimientos generales, incluyendo, en consecuencia: la creatividad, el talento, el entusiasmo, la motivación, las destrezas en la tarea, etc., es decir motivando el desarrollo' tanto de estrategias de Aprendizaje.. (Arancibia y Col. 1999) .como de su inteligencia emocional (García y Magaz,; 1982). En este contexto, Estrategias de Aprendizaje se refiere a aquellas habilidades para aprender que se utilizan en' la resolución de los problemas académicos cotidianos. y la Inteligencia Emocional se refiere a aquel subconjunto de la inteligencia social que comprende la capacidad de controlar los sentimientos y emociones propios así como los de los demás, de discriminar entre ellos y dc utilizar esta información para guiar el pensamiento y las acciones (Salovey y Mayer, 1990, citado por Shapiro. 1997).

Cuando se combinan los diferentes niveles de cohesión y adaptabilidad descritos por el Modelo Circumplejo de Olson se obtienen tres tipos de familias:

Balanceada, Rango medio y Extremo que dan lugar a distintos climas familiares, los mismos que se han descrito líneas arriba en la figura 1.

Los Objetivos que se buscaron en el presente estudio fueron:

a) Describir la relación entre el clima familiar (Balanceado, Rango Medio y Extremo) y las estrategias de aprendizaje.

b) Describir la asociación entre el clima familiar (Balanceado. Rango Medio' y Extremo) y la inteligencia emocional.

c) Describir la relación entre el clima familiar (Balanceado. Rango Medio y Extremo) y el rendimiento académico.

d) Describir y analizar los efectos combinados del clima familiar. los estilos de aprendizaje y la inteligencia emocional sobre el rendimiento académico.

En consecuencia, se estarían analizando las siguientes variables:

Variables Independientes:

Clima Familiar, Estilos de Aprendizaje e Inteligencia Emocional.

Variables Dependientes:

Rendimiento Académico. Variables Controladas:

Nivel Socio-económico: Medio.

Edad: 16-26. Sexo: Varones y Mujeres. Procedencia: Estudiantes de Psicología de la U.N.M.S.M.

Y sometiendo a contrastación las siguientes hipótesis de trabajo:

H1: El tipo de Clima Familiar percibido por el estudiante universitario (Balanceado, Rango Medio y Extremo) establece diferencias en sus estrategias de aprendizaje.

H2: Existirán diferencias en la inteligencia emocional al compararse a un grupo de estudiantes universitarios que se desenvuelven dentro de un clima familiar Balanceado, Rango Medio y Extremo.

H3: Existirán diferencias en el rendimiento académico al compararse a un grupo de 
estudiantes universitarios cuyo clima familiar es Balanceado, Rango Medio o Extremo,

H4: El tipo de familia (Balanceada, Rango Medio y Extrema), las estrategias de aprendizaje y la inteligencia emocional tendrán efectos combinados sobre el rendimiento académico.

\section{MÉTODO}

El presente es un estudio de carácter ex post facto de tipo correlacional.

\section{Muestra:}

Es de carácter no probabilística intencional y los datos se basarán en las respuestas a las diferentes pruebas psicológicas de 250 estudiantes de la Facultad de Psicología de la U.N.M.S.M.

\section{Instrumentos:}

Se han aplicado los siguientes instrumentos:

Ficha demográfica: Cuestionario estructurado de alternativas de respuesta cerradas. que permiten registrar algunas características relevantes de los sujetos estudiados: Edad, sexo, grado de instrucción, etc.

Cuestionario Fases III: Desarrollado por Olson, Sprenkle y Russell, con el objetivo de obtener una perspectiva del, interior del funcionamiento familiar tal como es percibido por diversos miembros de la familia. El cual esta conformado por una escala de evaluación de la adaptabilidad y otra de la cohesión Familiar. Traducido y validado en Lima por Malamut, quien halla un índice de correlación de Pearson entre las mitades de 0.58 para cohesión y de 0.54 para adaptabilidad. Además del índice dc consistencia interna de Spearman-Brown, donde se obtuvo 0.73 para cohesión y de 0.70 para adaptabilidad. Por otro lado se encontró un coeficiente al fa de Cronbach de 0.74 para cohesión y de 0.63 para adaptabilidad. Esta prueba evalúa los diferentes tipos de sistemas familiares de acuerdo al Modelo Circumplejo de David Olson el cual a partir de la combinación de cuatro niveles de Cohesión con cuatro niveles de adaptabilidad familiar permite identificar dieciséis tipos de sistemas maritales y tres tipos de familias, a saber:

Balanceada, Rango medio y Extrema. Se aplica a partir de los 12 años.

La Adaptabilidad familiar examina el grado en que el sistema familiar es flexible y capaz de cambiar. Se define como la habilidad de un sistema marital o familiar para cambiar su estructura dc poder, las relaciones de roles y las reglas que rigen las relaciones, en respuesta al estrés situacional y propio del desarrollo. Los conceptos específicos para medir y diagnosticar esta dimensión son: autoridad dentro de la familia (liderazgo, asel1ividad, control y disciplina), estilos de negociación, relaciones entre roles y reglas que rigen las relaciones.

Los grados de adaptabilidad oscilan entre rígida, estructurada, flexible y caótica:

Rígida: se caracteriza por el liderazgo autoritario y autocrático, disciplina estricta, rígida y de aplicación severa, los padres imponen decisiones, las reglas se hacen cumplir estrictamente no habiendo posibilidad de cambio y con roles estrictamente definidos. Estructurada: se caracteriza por ser democrática, los padres toman decisiones, siendo la disciplina rara vez severa y predecibles sus consecuencias, los roles son estables pero pueden comparlirse, las reglas se hacen cumplir firmemente y pocas son las que cambian, cl principio del liderazgo es autoritario, siendo algunas veces igualitario. Flexible: se caracteriza por un liderazgo igualitario y permitir cambios, la disciplina rara vez es severa y 
sus consecuencias son predecibles, usualmente es democrática y hay acuerdo en las decisiones, se comparten los roles y las reglas se hacen cumplir con flexibilidad, algunas reglas cambian. Caótica: se caracteriza por un liderazgo limitado e ineficaz, disciplina poco severa e inconsistente, falta de claridad en los roles, decisiones parentales impulsivas y frecuentes cambios en las reglas que se hacen cumplir inconsistentemente.

El Modelo Circumplejo postula que los niveles de adaptabilidad estructurado y flexible facilitan el funcionamiento conyugal y familiar, mientras que los extremos, rígido y caótico, resultan problemáticos para las familias.

La Cohesión familiar examina el grado en que los miembros de la familia están compenetrados y se ayudan y apoyan entre sí. Se define como el vínculo emocional que los miembros de la familia mantienen entre ellos. Los conceptos específicos para medir y diagnosticar esta dimensión son: vinculación emocional, dependencia, límites, coaliciones parento filiales, tiempo, espacio, amigos, toma de decisiones, intereses y recreación.

Los grados de cohesión oscilan entre desligada, separada, conectada y aglutinada.

Desligada: se caracteriza por la extrema separación emocional entre sus miembros, poca interacción, falta de cercanía parento filial, con predominio de la separación personal preferencia de espacios separados, intereses desiguales localizados fuera de la familia. Separada; se caracteriza por la separación emocional pero en ocasiones se demuestra la correspondencia afectiva, aceptar el involucramiento pero preferir la distancia, límites parento filiales claros, se considera impollante el tiempo individual pero pasan pm1e del tiempo juntos, se prefieren los espacios separados pero comparten el espacio familiar, las decisiones se toman individualmente pero hay posibilidad de decisiones conjuntas, interés focal izado fuera de la familia, la recreación se lleva a cabo más separada que compartida. Conectada: se caracteriza por la cercanía emocional, las interacciones afectivas son alentadas y preferidas, la lealtad familiar es esperada, el interés se focaliza dentro de la familia, enfatiza la interrelación pero se permite la distancia personal, la necesidad de separación es respetada pero no valorada, los límites entre los subsistemas son claros con cercanía parento filial, los amigos individuales y preferencias se comparten.

Aglutinada: se caracteriza por el involucramiento simbiótico, dependencia entre sus miembros, expresada con alta reactividad emocional, permiten poco tiempo y espacio privado, interés focalizado en la familia, hay coaliciones parento filiales.

El Modelo Circumplejo postula que los niveles de cohesión separada y conectada, facilitan el funcionamiento familiar, mientras que los extremos, desligada y aglutinada, resultan problemáticos para las familias.

El tipo de familia Balanceado, de acuerdo al Modelo Circumplejo, es el más adecuado y corresponde a las familias resultantes de la combinación de los siguientes grados de adaptabilidad y cohesión: flexiblemente separada, flexiblemente conectada, estructuralmente separada y estructuralmente conectada; FS, FC, ES y EC, respectivamente.

El tipo de familia Rango Medio corresponde a las familias resultantes de la combinación de los siguientes grados de adaptabilidad y cohesión: caóticamente separada, caóticamente conectada, flexiblemente desligada, flexiblemente aglutinada, estructuralmente desligada, estructural mente aglutinada, rígidamente separada y rígidamente conectada; CS, CC, FD, FA, ED, EA, RS y RC respectivamente.

El tipo de familia Extremo de acuerdo al Modelo Circumplejo es el menos adecuado y corresponde a las familias resultantes de la combinación de los siguientes grados de adaptabilidad y cohesión: caóticamente desligada, caóticamente aglutinada, rígidamente 
desligada y rígidamente aglutinada, $\mathrm{CD}, \mathrm{CA}, \mathrm{RD}$ y $\mathrm{RA}$, respectivamente.

Test de Inteligencia Emocional de Baron: Elaborado por Reuven Baron con la denominación original de EQ-I (Emotional Quotient Inventory), traducido y revisado en el Perú por Zoila Abanto, Leonardo Higueras y Jorge Cueto. Test de administración individual y colectiva, aplicable a sujetos mayores de 16 años, que permite evaluar las aptitudes emocionales de la personalidad determinantes para alcanzar el éxito general y mantener una salud emocional positiva. Incluye 133 ítemes con cinco alternativas de respuesta cada uno, requiriendo su administración entre 30 a 40 minutos, genera resultados de tres escalas de validez, un resultado de Cociente Emocional total, resultados de cinco escalas compuestas, a saber: Intrapersonal, interpersonal, adaptabilidad, manejo de la tensión y estado de ánimo general; además de los resultados de quince subescalas del Cociente Emocional. Su confiabilidad ha sido obtenida por el método de consistencia interna utilizando el estadístico Alfa de Cronbach obteniendo coeficientes para las quince subescalas que mide entre 0.69 y 0.86 , con un coeficiente de consistencia interna total de 0.76 , y por el método del test retest obteniendo un coeficiente de confiabilidad promedio para las quince subescalas en el retest después de un mes de 0.85 y después de cuatro meses de 0.75. Se han realizado nueve tipos de estudios de validez: Validez aparente y de contenido (sí los itemes parecían estar capturando la esencia de cada escala y subescala). Validez de factores (el grado en el cual la estructura original del cuestionario es confirmada), Validez de construcción, convergente, divergente y de criterio de grupo (el grado en que las escalas están midiendo lo que se supone deben medir en vez de algo más). validez discriminante (el grado en el cual las subescalas permiten identificar y diferenciar entre personas que son emocional mente más inteligentes de las que son emocional mente menos inteligentes) y validez predecible (si el test permite predecir la conducta emocional mente inteligente en el futuro), encontrando en todos los casos coeficientes de validez claramente adecuados, por lo cual aquellos que administren este cuestionario pueden, con toda seguridad, con fiar en la precisión y calidad de los resultados que obtendrán. (Abanto, Higueras y Cueto, 2000).

Escala de Estrategias de Aprendizaje (ACRA): Desarrollada por Roman y Gallego (1992) con el objetivo de obtener una evaluación o diagnóstico previo a la intervención. Aplicable a partir de los 12 años, en forma individual o colectiva, sin tiempo limitado, pero su aplicación completa suele durar unos 50 minutos, Puntuación del item: de uno a cuatro, rango de aplicación de 12 a 16 años pudiendo ser ampliada a universitarios. Esta compuesta de cuatro subescalas independientes que evalúan el uso que habitualmente hacen los estudiantes de siete estrategias de Adquisición de información, de trece estrategias de Codificación de información, de cuatro estrategias de Recuperación de información y de nueve estrategias de Apoyo al procesamiento de la información.

Los procesos cognitivos de Adquisición de información son los procesos encargados de seleccionar, transformar y transportar la información desde el ambiente al Registro Sensorial y de este a la Memoria a Corto Plazo (MCP), aquí se constatan estrategias que favorecen el control y dirección de la atención, y aquellas que optimizan los procesos de repetición.

Los procesos cognitivos de Codificación de información son los procesos encargados de transformar y transportar la información de la MCP a la Memoria a Largo Plazo (MLP). La elaboración (superficial o profunda) y la organización más sofisticada de la información, conectan esta con los conocimientos previos integrándola en estructuras de significado más amplias (formas de representación) que constituyen la llamada estructura cognitiva o base de conocimientos. Aquí se constatan tres grupos de estrategias, a saber: de 
Nemotecnización, de elaboración y de organización.

Los procesos cognitivos de Recuperación de información son los procesos encargados de transformar y transportar la información desde la estructura cognitiva a la MCP, son aquellos que favorecen la búsqueda de información en la memoria y la generación de Respuestas; dicho de otra manera, aquellos que le sirven para manipular (Optimizar) los procesos de recuperación o recuerdo mediante sistemas de búsqueda o de generación de respuestas.

Los procesos de Apoyo al procesamiento de la información son los procesos de naturaleza meta cognitivas o no-cognitiva que optimizan, son neutrales o entorpecen el funcionamiento de las estrategias cognitivas de aprendizaje, cuando estos procesos optimizan el rendimiento de las estrategias dc adquisición, codificación y recuperación, incrementan la motivación, la autoestima, la atención. Garantizando el clima adecuado para un buen funcionamiento de todo el sistema cognitivo. Incluyen las llamadas estrategias meta cognitivas y las socio afectivas.

Se han obtenido cuatro tipos de Validez, a saber: Validez de contenido con el coeficiente de correlación de pearson, Obteniendo los siguientes índices: adquisición 0.87, codificación 0.89 , recuperación 0.91 y apoyo 0.88 , Validez de constructo por el método del juicio de expertos, pearson, adquisición 0.87 , codificación 0.87 , recuperación 0.89 y apoyo 0.88 , Validez predictiva con las asignaturas de ciencias, historia, lengua y matemática y además la Validez factorial. En cuanto a la fiabilidad, han sido obtenidos dos índices: El coeficiente alfa, con correlaciones dc: adquisición 0.71 , codificación 0.90 , recuperación 0.84 y apoyo 0.90, el de pares - impares con las correlaciones de spearman de: adquisición 0.61, codificación 0.80 , recuperación 0.72 y apoyo 0.73 .

\section{Rendimiento Académico:}

Estimado a partir del promedio ponderado de las calificaciones obtenidas por los alumnos durante su permanencia en la Universidad, extraídas de los registros existentes en la oficina de matrícula respectiva del record académico del estudiante.

\section{Procedimientos:}

Luego de establecer las coordinaciones necesarias se efectuó un muestreo al azar, extrayéndose años de estudios como muestras representativas de los alumnos de la Facultad. Se recogió la información evaluando a las promociones seleccionadas. Efectuando luego el depurado de los datos y la elaboración de la base de datos. Sometiendo, por último, los datos al análisis y procesamiento estadístico en la aplicación SPSS FN 9.0.

\section{Resultados y Discusión}

En cuanto al comportamiento de las variables en la muestra encontramos: Los estudiantes investigados presentan un promedio ponderado de rendimiento académico de 14.8, el cual si bien se acompaña de un puntaje modal de 14.5, si embargo se distribuye según la curva Normal (ver Gráfico 1), oscilando la mayoría de los puntajes entre 12.5 y 17.5.

En todas las estrategias de aprendizaje evaluadas por el test de ACRA, estos muchachos se encuentran en el eneatipo 5. es decir dentro de un rango promedio (Grafico 2).

En cuanto a su Inteligencia Emocional, estos sujetos presentan un nivel prácticamente promedio en los componentes interpersonales, de manejo del estrés y del estado de animo general, una ligera baja en el nivel del componente de adaptabilidad y prácticamente una capacidad emocional por debajo del promedio respecto al componente Intrapersonal, es decir las habilidades para comprender, controlar y desarrollar las propias emociones (Grafico 3). Esto es confirmado por los resultados observados en el grafico 4, donde 
podemos constatar que el $65 \%$ de los sujetos de la muestra se encuentran con una capacidad emocional adecuada o promedio, sólo el $34 \%$ necesita mejorar su habilidad emocional, e incluso se encuentra un $1 \%$ que presenta una capacidad emocional muy desarrollada

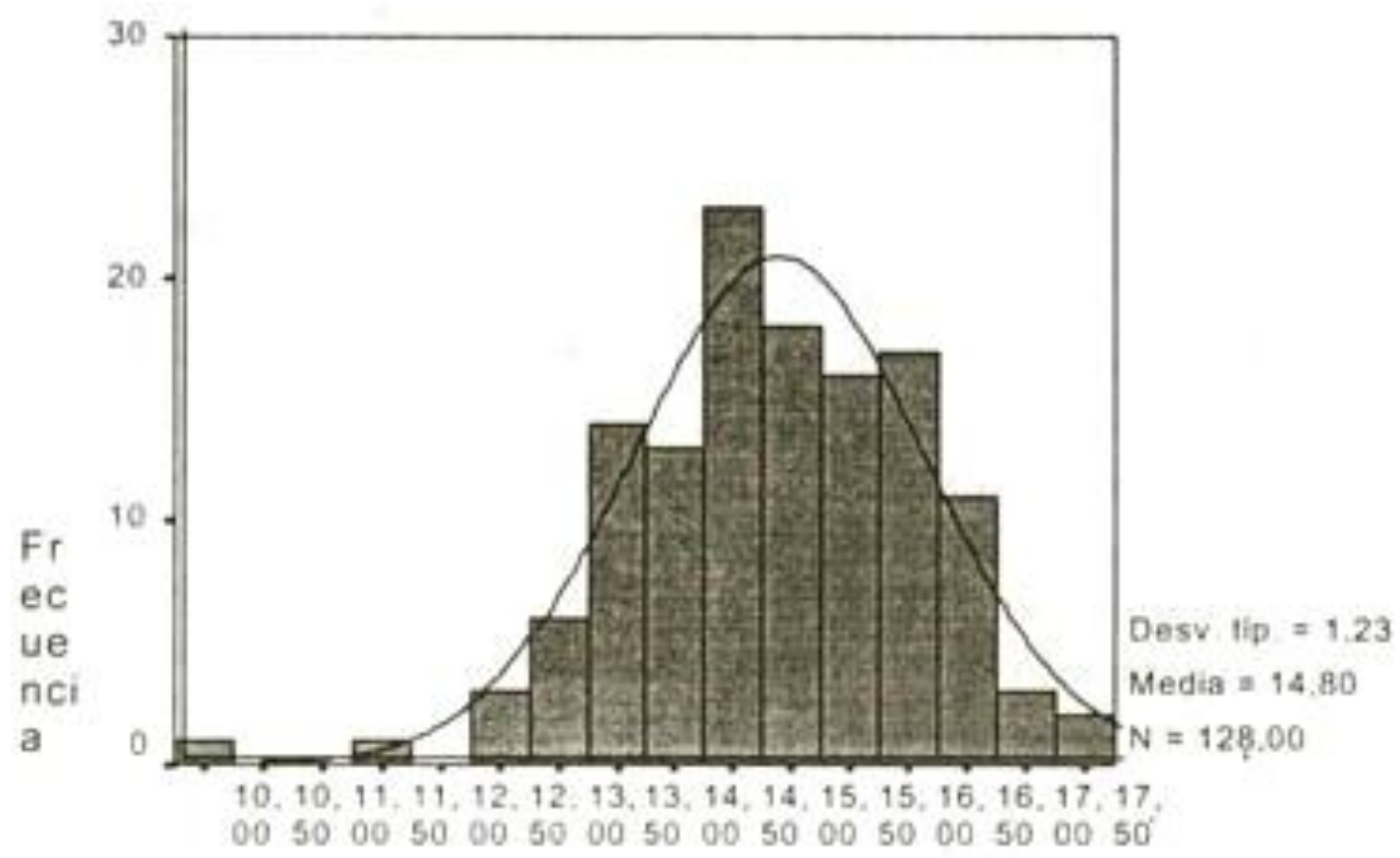

PromedioPonderado

Grafico 1.- Promedio de Rendimientos Académico

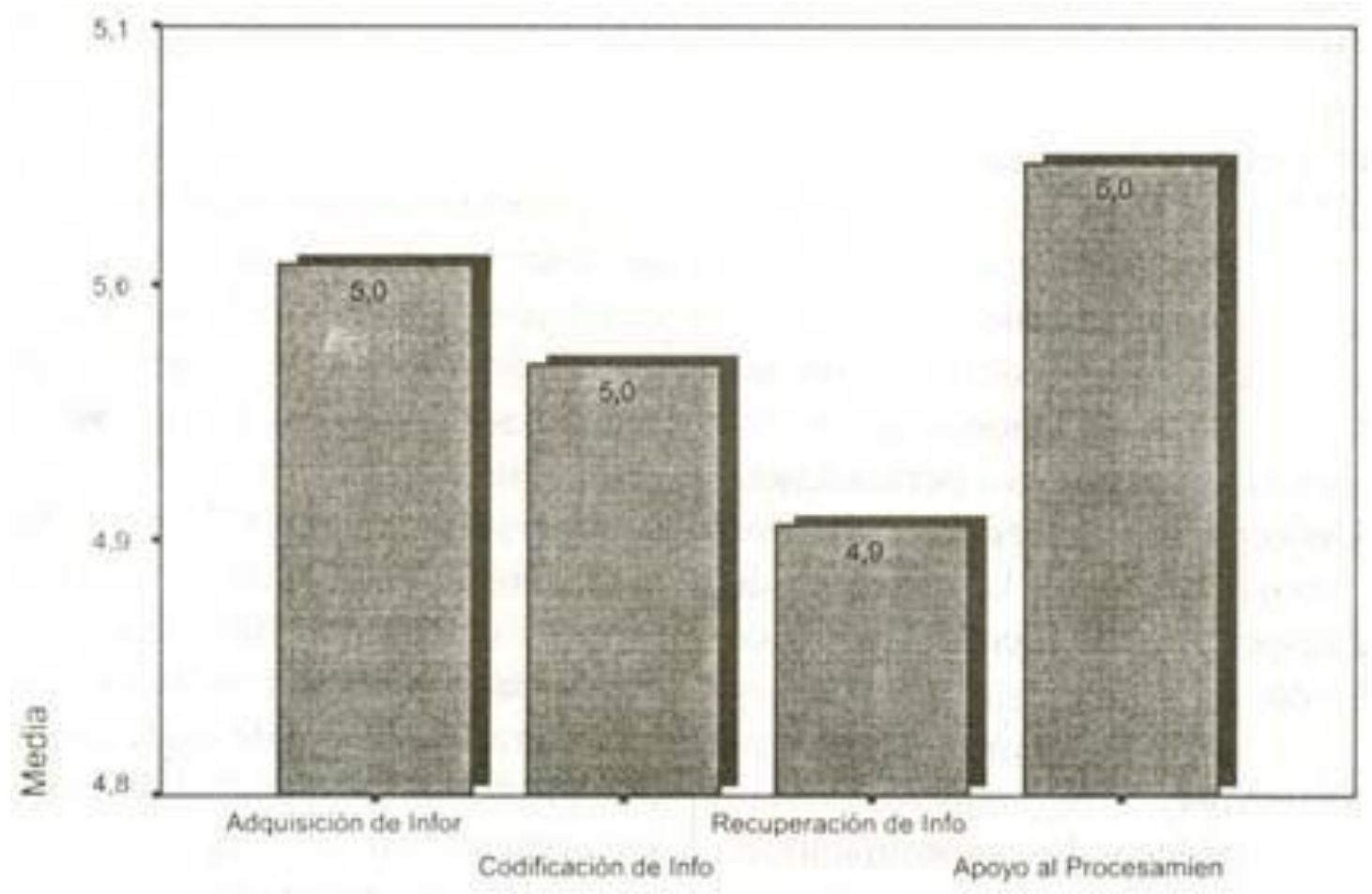


Grafico 2.- Distribución de las estrategias de aprendizaje en la muestra

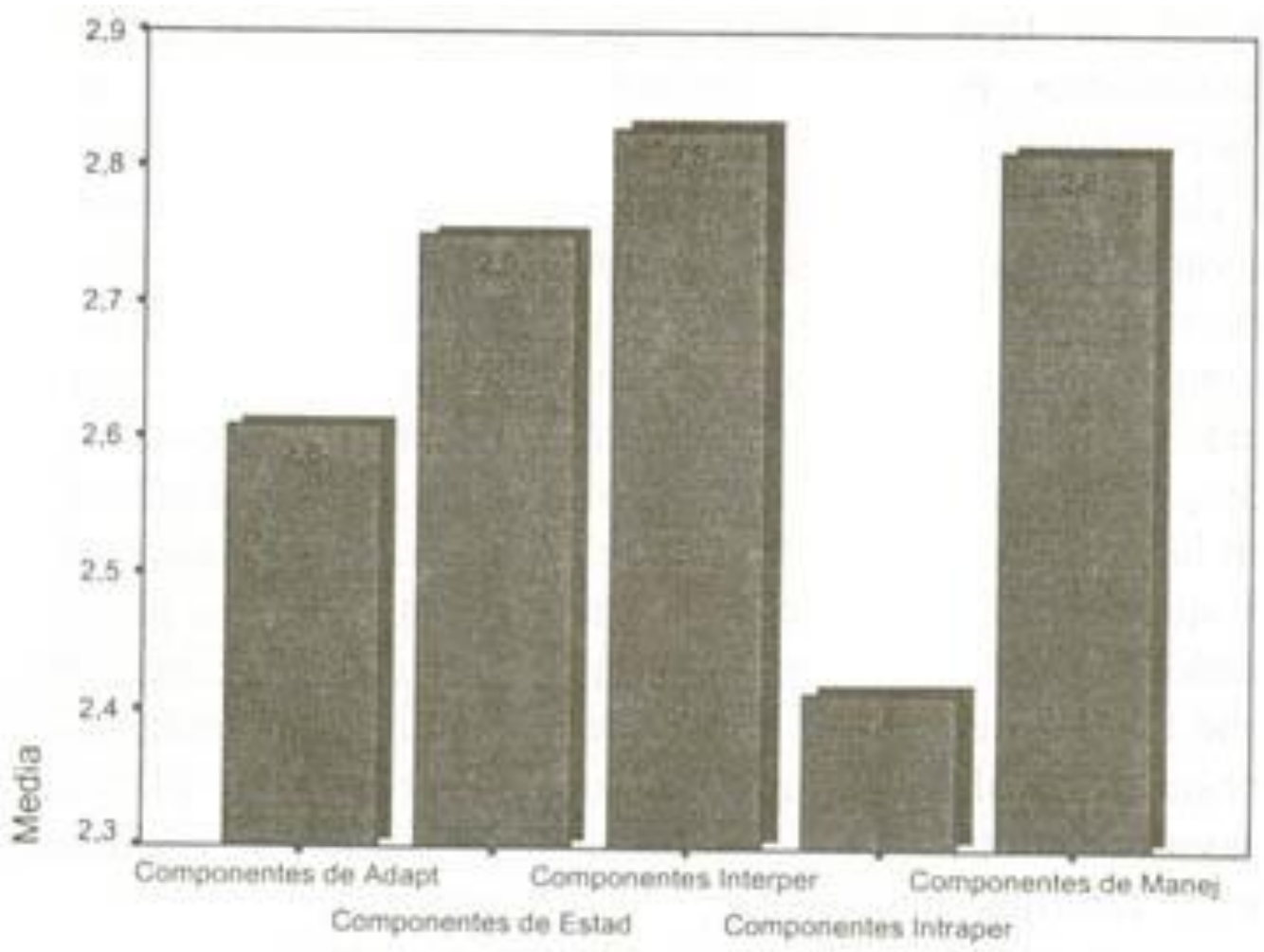

Grafico 3.- Distribución de los componentes del C.E.

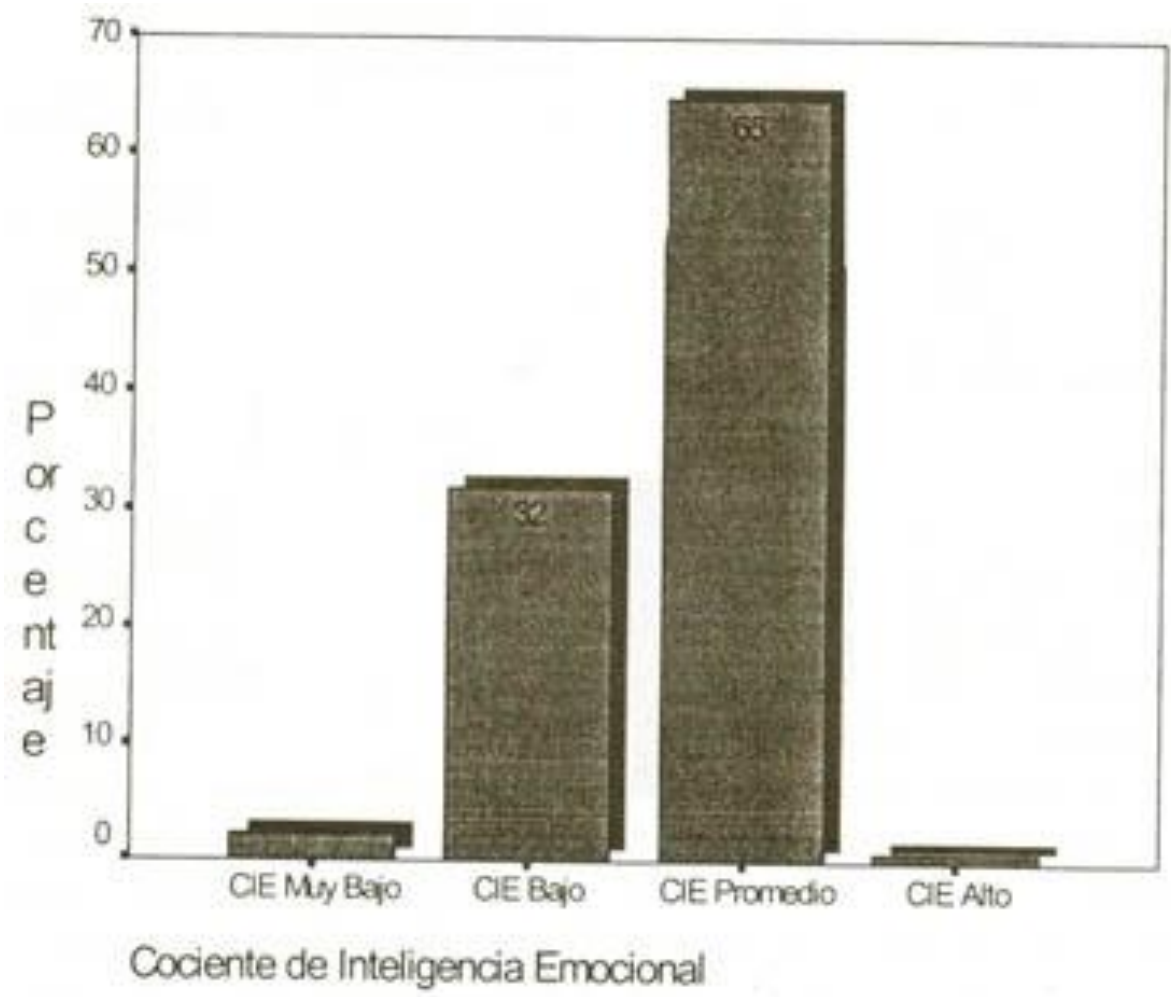




\section{Grafico 4.- Cociente Emocional General}

En relación, al tipo de familia que nuestros estudiantes presentan, identificadas por la combinación de los niveles de cohesión (lazos emocionales, limites familiares, capacidad de tomar decisiones, coaliciones, tiempo, espacio, intereses y amigos compartidos) y adaptabilidad (Asertividad, liderazgo, disciplina, negociación, roles y reglas) que ellos perciben en las mismas, constatamos en el Grafico 5 que el $83.6 \%$ percibe a su familia como Promedio o balanceada, mientras que tan sólo el $16.4 \%$ la percibe como extrema, lo cual indica que en su gran mayoría nuestros alumnos procederían de familias funcionales.

En relación a nuestras hipótesis de trabajo encontramos que los hallazgos respecto a las estrategias de aprendizaje evaluadas por el test de ACRA (Adquisición, codificación, recuperación y apoyo al procesamiento de la información), se encuentran en un rango promedio.(ver gráfico 6)

Sin embargo, es evidente que los alumnos provenientes de Familias Balanceadas destacan en las estrategias de aprendizaje hasta en dos eneatipos por encima de los provenientes de Familias extremas, coincidiendo con nuestros postulados, resultados que se confirman la "Prueba t", (Tabla 1), en el cual encontramos diferencias significativas para todas las estrategias de aprendizaje evaluadas a excepción de la estrategia de Recuperación de Información

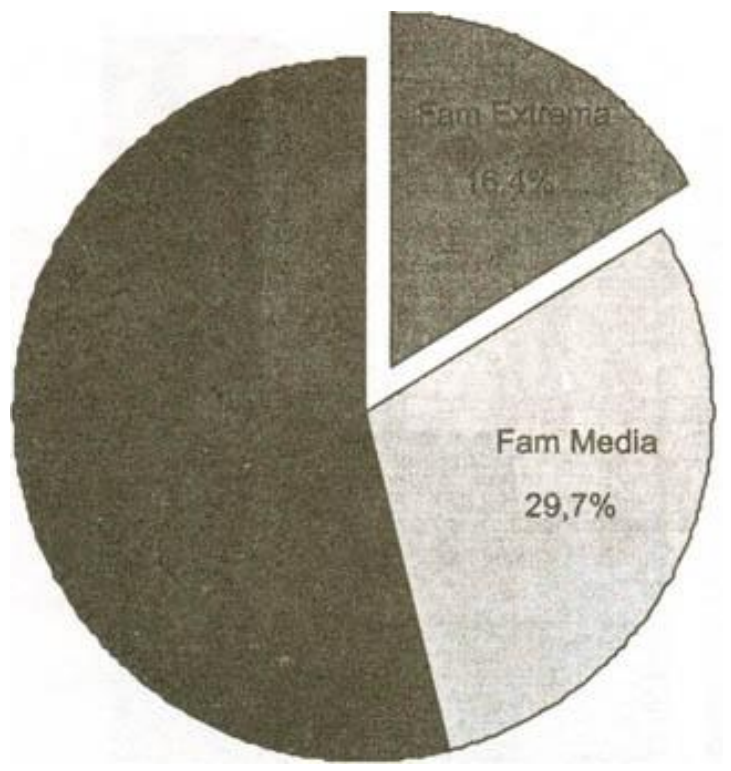

Gráfico 5.- Distribución de los tipos de Familia en la Muestra 


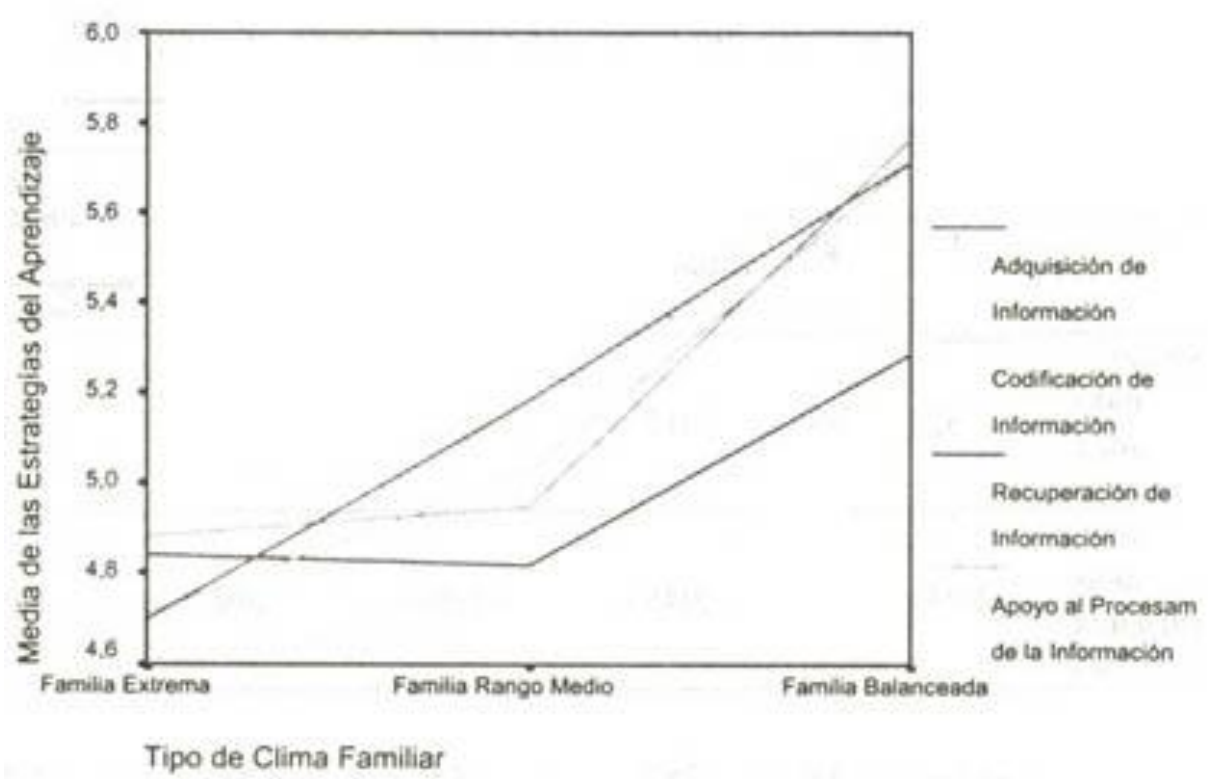

Grafico 6.- Promedio de Estrategias de Aprendizaje por cada Tipo de Familia

Tabla 1.- Prueba $t$ de las estrategias de aprendizaje comparando familias balanceadas versus extremas

\begin{tabular}{clccc}
\hline & $\begin{array}{c}\text { Tipo de Clima } \\
\text { Familiar }\end{array}$ & $\mathrm{N}$ & Media & $\begin{array}{c}\text { Desviación } \\
\text { tip. }\end{array}$ \\
\hline Adquisición de & Familia Extrema & 69 & 4.70 & .19 \\
Información & $\begin{array}{l}\text { Familia } \\
\text { Balanceada }\end{array}$ & 21 & 5.71 & .38 \\
\hline Codificación de & Familia Extrema & 69 & 4.71 & .23 \\
Información & Familia & 21 & 5.71 & .50 \\
& Balanceada & & & \\
\hline Recuperación de & Familia Extrema & 69 & 4.84 & .22 \\
Información & Familia & 21 & 5.29 & .20 \\
& Balanceada & & & \\
\hline Apoyo al & Familia Extrema & 69 & 4.88 & .20 \\
Procesamiento & Familia & 21 & 5.76 & .40 \\
& Balanceada & & & \\
\hline
\end{tabular}

Respecto a la segunda hipótesis de trabajo, a saber H2: Existirán diferencias en la inteligencia emocional al compararse a un grupo de estudiantes universitarios que se desenvuelven dentro de un clima familiar Balanceado, Rango Medio y Extremo, los datos obligan a aceptar que sólo parcialmente resulta cierta, puesto que podemos observar (Grafico 7) que tanto en el cociente de inteligencia emocional general, como en tres de sus cinco componentes, prácticamente no se presentan diferencias en cuanto al nivel de sus capacidades entre los tres tipos de familias, lo que se corrobora con los resultados de la "Prueba t" que al comparar los componentes de la inteligencia emocional en las familias de tipo Balanceado y Extremo, solamente encuentra diferencias significativas en los componentes de adaptabilidad y de estado de animo general (Tabla 2) . 
Tabla 1.- Prueba t de las estrategias de aprendizaje comparando familias balanceadas versus extremas

Prueba T para la igualdad de medias

$\mathrm{t}$ gl Sig. Diferencia Error tip. Intervalo de
(bilateral) de medias de la confianza para la diferencia media Inferior Superior

\begin{tabular}{|c|c|c|c|c|c|c|c|c|}
\hline $\begin{array}{l}\text { Adquisición } \\
\text { de } \\
\text { Información }\end{array}$ & $\begin{array}{l}\text { Se han } \\
\text { asumido } \\
\text { varianzas } \\
\text { iguales }\end{array}$ & 2.523 & 88 & .013 & -1.02 & .40 & -1.82 & -22 \\
\hline $\begin{array}{l}\text { Codificación } \\
\text { de } \\
\text { Información }\end{array}$ & $\begin{array}{l}\text { Se han } \\
\text { asumido } \\
\text { varianzas } \\
\text { iguales }\end{array}$ & 2.031 & 88 & .045 & -1.00 & .49 & -1.99 & $\begin{array}{c}-2.1 \mathrm{E}- \\
02\end{array}$ \\
\hline $\begin{array}{l}\text { Recuperación } \\
\text { de } \\
\text { Información }\end{array}$ & $\begin{array}{l}\text { Se han } \\
\text { asumido } \\
\text { varianzas } \\
\text { iguales }\end{array}$ & 1.083 & 88 & .282 & -.45 & .41 & -1.26 & .37 \\
\hline $\begin{array}{l}\text { Apoyo al } \\
\text { Procesamiento }\end{array}$ & $\begin{array}{l}\text { Se han } \\
\text { asumido } \\
\text { varianzas } \\
\text { iguales }\end{array}$ & $\begin{array}{c}- \\
2.086\end{array}$ & 88 & .040 & -.88 & .42 & -1.71 & $\begin{array}{c}-4.2 \mathrm{E}- \\
02\end{array}$ \\
\hline
\end{tabular}

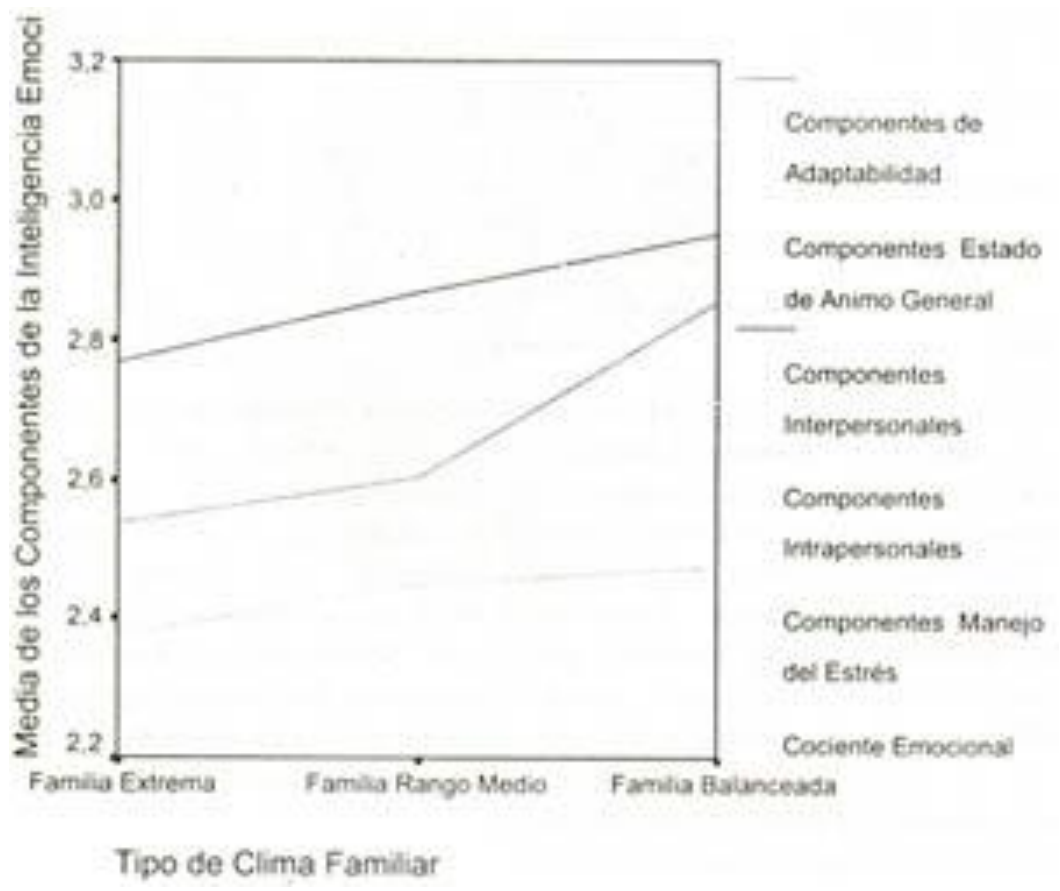

Grafico 7- Promedio de los componentes de la Inteligencia Emocional por los tipos de Familia 
Tabla 2- Prueba t de los componentes de la Inteligencia emocional comparando familias balanceadas versus extremas

\begin{tabular}{cllcc}
\hline & $\begin{array}{c}\text { Tipo de Clima } \\
\text { Familiar }\end{array}$ & N & Media & Desviación tip. \\
\hline Componentes de & Familia Extrema & 69 & 2.54 & .56 \\
Adaptabilidad & Familia Balanceada & 21 & 2.86 & .65 \\
\hline Componentes de Estado & Familia Extrema & 69 & 2.65 & .59 \\
de Animo General & Familia Balanceada & 21 & 3.10 & 1.00 \\
\hline Componentes & Familia Extrema & 69 & 2.77 & .77 \\
Interpersonales & Familia Balanceada & 21 & 2.95 & 1.07 \\
\hline Componentes Intra & Familia Extrema & 69 & 2.38 & .60 \\
personales & Familia Balanceada & 21 & 2.48 & .68 \\
\hline Componentes de manejo & Familia Extrema & 69 & 2.80 & .65 \\
del estrés & Familia Balanceada & 21 & 3.10 & .77 \\
\hline Cociente de Inteligencia & Familia Extrema & 69 & 2.60 & .53 \\
Emocional & Familia Balanceada & 21 & 2.71 & .64 \\
\hline
\end{tabular}

Tabla 2.- Prueba t de los componentes de la Inteligencia emocional comparando familias balanceadas versus extremas

Prueba T para la igualdad de medias

\begin{tabular}{|c|c|c|c|c|}
\hline gl & $\begin{array}{c}\text { Sig. } \\
\text { (bilateral) }\end{array}$ & $\begin{array}{l}\text { Diferencia } \\
\text { de medias }\end{array}$ & $\begin{array}{l}\text { Error tip. } \\
\text { de la } \\
\text { diferencia }\end{array}$ & $\begin{array}{c}\text { Intervalo de } \\
\text { confianza para la } \\
\text { media }\end{array}$ \\
\hline
\end{tabular}

\begin{tabular}{|c|c|c|c|c|c|c|c|c|}
\hline $\begin{array}{l}\text { Componentes de } \\
\text { Adaptabilidad }\end{array}$ & $\begin{array}{l}\text { Se han } \\
\text { asumido } \\
\text { varianzas } \\
\text { iguales }\end{array}$ & -2.215 & 88 & .029 & -.32 & .14 & -.61 & $-3.3 \mathrm{E}-02$ \\
\hline $\begin{array}{l}\text { Componentes de } \\
\text { Estado de Animo } \\
\text { General }\end{array}$ & $\begin{array}{l}\text { Se han } \\
\text { asumido } \\
\text { varianzas } \\
\text { iguales }\end{array}$ & -2.530 & 88 & .013 & -.44 & .18 & -.79 & $-9.5 \mathrm{E}-02$ \\
\hline $\begin{array}{l}\text { Componentes } \\
\text { Interpersonales }\end{array}$ & $\begin{array}{l}\text { Se han } \\
\text { asumido } \\
\text { varianzas } \\
\text { iguales }\end{array}$ & -.872 & 88 & .385 & -.18 & .21 & -.60 & .24 \\
\hline $\begin{array}{l}\text { Componentes } \\
\text { Intra personales }\end{array}$ & $\begin{array}{l}\text { Se han } \\
\text { asumido } \\
\text { varianzas } \\
\text { iguales }\end{array}$ & -.647 & 88 & .519 & $-9.94 \mathrm{E}-02$ & .15 & -.40 & .21 \\
\hline $\begin{array}{l}\text { Componentes de } \\
\text { manejo del estrés }\end{array}$ & $\begin{array}{l}\text { Se han } \\
\text { asumido } \\
\text { varianzas } \\
\text { iguales }\end{array}$ & -1.753 & 88 & .083 & -.30 & .17 & -.64 & $3.98 \mathrm{E}-02$ \\
\hline $\begin{array}{l}\text { Cociente de } \\
\text { Inteligencia } \\
\text { Emocional }\end{array}$ & $\begin{array}{l}\text { Se han } \\
\text { asumido } \\
\text { varianzas } \\
\text { iguales }\end{array}$ & -.666 & 88 & .507 & $-9.11 \mathrm{E}-02$ & .14 & -.36 & .18 \\
\hline
\end{tabular}


En relación a la H3: Existirán diferencias en el rendimiento académico al compararse a un grupo de estudiantes universitarios cuyo clima familiar es Balanceado, Rango Medio o Extremo, se acepta la hipótesis nula de que no se encuentran diferencias estadísticamente significativas entre los tres tipos de familia en cuanto al rendimiento académico de los alumnos, estimado por su promedio ponderado.

En cuanto a nuestra ultima hipótesis, de si el tipo de familia ( Balanceada, Rango Medio o Extrema), los estilos de aprendizaje y la inteligencia emocional tendrían efectos combinados sobre el rendimiento académico, si observamos la tabla 3, podemos constatar correlaciones significativas incluso al nivel 0 de significación de 0.01 , entre las estrategias de aprendizaje, el cociente de inteligencia emocional e incluso entre dos de las estrategias de aprendizaje y el tipo de Clima Familiar, pero no así entre estas variables y el promedio de rendimiento académico, el cual por el contrario presenta correlaciones más bien negativas con las otras variables, mismas que sin embargo no llegan a ser significativas. En tanto las variables estudiadas a excepción del Promedio académico son medidas en escala Ordinal, no resulta aconsejable efectuar una regresión lineal, así como tampoco un ANOV A, puesto que no se podrían asegurar las condiciones que estos estadísticos requieren, en consecuencia en este caso también tendríamos que aceptar la hipótesis nula de que los tipos de familia, estrategias de aprendizaje y cociente de inteligencia emocional no dan cuenta significativamente del comportamiento del rendimiento académico de los estudiantes en la muestra estudiada.

Tabla 3.- Correlaciones Rho de Spearman de las Estrategias de Aprendizaje , el Cociente de Inteligencia Emocional, El Tipo de Clima Familiar y el promedio Académico

\begin{tabular}{|c|c|c|c|c|c|c|}
\hline & $\begin{array}{l}\text { Adquisición } \\
\text { de } \\
\text { Información }\end{array}$ & $\begin{array}{l}\text { Codificación } \\
\text { de } \\
\text { Información }\end{array}$ & $\begin{array}{l}\text { Recuperación } \\
\text { de } \\
\text { Información }\end{array}$ & $\begin{array}{c}\text { Apoyo al } \\
\text { Procesamiento }\end{array}$ & $\begin{array}{c}\text { Cociente } \\
\text { de } \\
\text { Inteligencia } \\
\text { Emocional }\end{array}$ & $\begin{array}{l}\text { Tipo de } \\
\text { Clima } \\
\text { Familiar }\end{array}$ \\
\hline $\begin{array}{l}\text { Codificación } \\
\text { de } \\
\text { Información }\end{array}$ & $.699 * *$ & & & & & \\
\hline $\begin{array}{l}\text { Recuperación } \\
\text { de } \\
\text { Información }\end{array}$ & $.560 * *$ & $.649 * *$ & & & & \\
\hline $\begin{array}{l}\text { Apoyo al } \\
\text { Procesamiento }\end{array}$ & $.513 * *$ & $.543 * *$ & $.568 * *$ & & & \\
\hline $\begin{array}{l}\text { Cociente de } \\
\text { Inteligencia } \\
\text { Emocional }\end{array}$ & $.187^{*}$ & $.241 * *$ & $.282 * *$ & $.253 * *$ & & \\
\hline $\begin{array}{l}\text { Tipo de Clima } \\
\text { Familiar }\end{array}$ & $.194 *$ & $.156^{*}$ & .063 & .142 & .058 & \\
\hline $\begin{array}{l}\text { Promedio } \\
\text { Ponderado }\end{array}$ & -.111 & -.087 & -.052 & -.093 & -.052 & .056 \\
\hline
\end{tabular}

** La correlación es significativa al nivel 0.01 (unilateral)

* La correlación es significativa al nivel 0.05 (unilateral) 


\section{CONCLUSIONES:}

Los hallazgos en cuanto a los tipos de familia, donde encontramos que predominan las familias de rango medio y balanceadas, representan una fortaleza en cuanto a los hogares de los cuales proceden nuestros estudiantes, la misma que tendría que ser aprovechada por las acciones de la OFAYOE, con el objetivo de desarrollar la inteligencia emocional, las estrategias para el aprendizaje y, en consecuencia, su rendimiento académico, que si bien se encuentran dentro de los rangos promedios, sin embargo si la facultad se propone impartir una enseñanza dentro de una cultura de Calidad, resulta necesario estimularlas.

Mención aparte requiere la capacidad emocional por debajo del promedio observada en los componentes intrapersonales, de la inteligencia emocional, la cual representa una debilidad de nuestros estudiantes, en tanto que tiene que representar un desafió para quienes participamos en su formación el superarla, puesto que no es posible concebir un perfil profesional de Psicólogo que no incluya entre sus competencias, un nivel al menos promedio en estos componentes.

Son de resaltar las correlaciones estadísticamente significativas encontradas entre los tipos de familia, la inteligencia emocional y las estrategias de aprendizaje puesto que estarían indicando la necesidad de establecer los principios teóricos que subyacen a esta verificación fáctica de su asociación, y abre el camino para proyectos de investigación que establezcan las relaciones causal es existentes entre ellas.

Por último queda pendiente para futuras investigaciones analizar si la extraña falta de relación del promedio ponderado de las calificaciones obtenidas por los alumnos durante su permanencia en la Universidad, incluso con una variable tan ligada teóricamente al rendimiento académico como las estrategias de aprendizaje quizás podría haberse debido a su pequeño rango de distribución en la población observada, o debido a que el mismo no es un indicador adecuado del rendimiento académico, o debido a algún otro factor aún por investigar.

\section{REFERENCIAS:}

Abanto, Z, Higueras, L. Cueto, J. (2000) ICE Inventario de Cociente Emocional de Bar On. Lima-Perú.

García, E. y Magaz, A. (1982) Ratones, Dragones y Seres Humanos Auténticos. CEPE. Madrid.

Benites, L., (1998). Tipos de Familia, Clima Social Familiar y Asertividad en Adolescentes. Test de Maestría USMP. Lima.

Olson, D., Portner, J., \& Bell, R. (1982). Family adaptability and cohesion evaluation scales. In D. Olson et al. Family inventories used in a national survey of families across the family life cycle (pp. 5-24) S1. Paul: Family Social Science, University of Minnesota.

Olson, D., Russell, C., \& Sprenkle, D. (1983). Circumplex model of marital and family systems: VI. Theoretical update. Family Process, 22, 69-83. New York:Oxford University Press.

Román, J. M. Y Gallego, S. (1992). Estrategias de procesamiento de información: clasificación y escala de evaluación. II Simposium de Psicología Evolutiva y Educativa. 1-4 de Abril. 
Shapiro, L.: (1997). La inteligencia emocional de los niños. Vergara. Buenos Aires.

Steinberg, L. \& Hill, J. (1978). Patterns of family interaction as a function of age, the onset of puberty and formal thinking. Developmental Psychology.14, 6, 683, 684. 